J. R. Strohecker, Darstellungsverfahren der Hainstädter Ceritthone. 775

bliebenen Reste saurer Milch auf die frische Füllung übertragen wird, ist also wahrscheinlich verschieden rom Kumys- und KefirPilze.

Um mich über die Gegenwart des Alkohols zu vergewissern, hatte ich eine kleine Flasche mit 30 Stunden alter Omeire gefüllt und zur Verhinderung weiterer Gährung mit Salicylsäure versetzt. Nach meiner Rückkehr aus Hereroland - es waren inzwischen drei Monate vergangen - destillirte ich die Flüssigkeit ab und bestimmte den Alkohol als Jodoform. Ich fand 0,007 Proc. Alkohol. Leider hatte ich nicht auch eine Probe frischer Omeire mitgenommen und kann daher nur die Vermuthung aussprechen, dass die frische Omeire wahrseheinlich mehr Alkohol enthält, dass derselbe aber bei dem Aufbewahren des Getränkes schon während des ersten Tages zu Essigsäure oxydirt wird.

\title{
Darstellungsverfahren für die seltenen Erden der Hainstädter Ceritthone.
}

\author{
Von Dr. Jones Rudolph Strohecker.
}

Nachdem die Untersuchung des Materials, welches durch die in neuerer Zeit eingeführte arzneiliche Verwendung des Ceroxyds das pharmaceutische Interesse auf sich lenkt, ihre, in meinen beiden ersten Abhandlungen (Journal f. pr. Chemie 1886. Seite 132 und 260) beschriebene, primitive Stufe passirt hatte, sowie zufolge Kritik einer eigenen Controlle unterworfen worden war (Keim, Technische Mittheilungen, München 1886, Nr. 23 u. 24, und Chemisches Centralblatt 1. September 1886) stellte sich die Ursache heraus, aus welcher in den meisten Fällen das Cer auf gewöhnliche Weise nicht ausgeschieden werden kann, und handelte es sich deshalb darum, ein wenigstens befriedigendes Darstellungsverfahren für die seltenen Erden der Ceritthone aufzufinden.

Die Ursache, aus welcher das Cer der Abscheidung, beziehungsweise sogar der Beobachtung gewöhnlich sich entzieht, ist Anwesenheit von $\mathrm{Fe}^{2} \mathrm{O}^{3}$; enthält der Ceritthon mehr als circa 0,5 Proc. desselben, so gewinnt man mittelst Oxalsäure und Kaliumsulfat nicht die Niederschläge, wie bei der Verarbeitung des Cerits, sondern eisenreiche. Das aus dem Alaunniederschlage mit KHO abgeschie- 
dene Oxyd ist lederbraun, welche Farbe es auch beim Glühen behält, und verhält sich als eine sehr innige Oxydverbindung von $\mathrm{Fe}$ und Ce, über deren Constitution bis jetzt nichts Näheres bekannt ist. Schlechthin scheint dieser Körper von manchem Chemiker „Eisenoxyd" genannt worden zu sein.

Es wurden deshalb sehr zahlreiche Trennungsversuche desselben vorgenommen, unter welchen eine Krystallisation von Cerosulfat vorkommt; es schossen nämlich aus einer schwefelsauren Auflösung eisenhaltigen „Hainstädter" Cers spitze Pyramiden an, die von mir und dann von einem Mitgliede der Berliner Universität geprüft worden sind. Da nun weitere beschleunigte Krystallisationsversuche eine zwar sehr reichliche Ausbeute, jedoch keine eisenfreien Krystalle ergaben, so musste ein rein chemisches Verfahren ausgesucht werden; für das brauchbarste habe ich folgendes gehalten.

Der rothe Ceritthon (Nr. 1) wird mit reiner $\mathrm{HCl}$ bis zur Entfärbung, der bituminöse gleich lange gekocht, das Filtrat zur Entfernung von $\mathrm{SiO}^{2}$ und $\mathrm{Al}^{2} \mathrm{O}^{3}$ - daneben ${ }^{\circ} \mathrm{BeO}$ - mit Aetzkali behandelt und der ausgewaschene Niederschlag (bestehend aus $\mathrm{Fe}^{2} \mathrm{O}^{3}$, $\mathrm{Ce}^{2} \mathrm{O}^{3}, \mathrm{LaO}$, eventuell $\mathrm{DiO}, \mathrm{YO}, \mathrm{CaO}, \mathrm{MgO}$ und Phosphaten) wieder in $\mathrm{HCl}$ gelöst und mit $\mathrm{KHS}$ (nicht $\left(\mathrm{NH}^{4}\right)^{2} \mathrm{~S}$ ) versetzt; hierbei geht $\mathrm{PO}^{4} \mathrm{H}^{3}$ in Lösung und die Erden fallen sämmtlich wieder nieder. Aus der erneuten salzsauren Auflösung wird mittelst Natriumacetats und Natriumhypochlorits alles $\mathrm{Fe}$ und $\mathrm{Ce}$ abgeschieden, während alle übrigen Erden in Lösung bleiben.

Der meist lederfarbige Oxydniederschlag von $\mathrm{Fe}$ und $\mathrm{Ce}$ wird in $\mathrm{HCl}$ gelöst, die Lösung mit überschüssigem $\mathrm{NH}^{4} \mathrm{Cl}$, um Cersalmiak zu bilden, stark gekocht und mit $\mathrm{SO}^{2}$ vollkommen reducirt, mit $\mathrm{NH}^{3}$ neutralisirt und mit $\left(\mathrm{NH}^{4}\right)^{2} \mathrm{~S}$ das $\mathrm{FeO}$ ausgefällt. Das Filtrat enthält Cersalmiak; dasselbe wird eingetrocknet und der Rückstand geglüht, wobei citronengelbes $\mathrm{Ce}^{3} \mathrm{O}^{4}$ (Atomg. 92,1) hinterbleibt, welches im Tiegel in seiner Farbe leuchtet. Das von Ce und Fè befreite, die übrigen Erden enthaltende Filtrat wird mit überschüssigem $\mathrm{NH}^{4} \mathrm{Cl}$ gekocht, um La-, $\mathrm{Y}$ - eventuell Di-Salmiak zu bilden, sodann $\mathrm{CaO}$ mit Ammoncarbonat entfernt und das weitere Filtrat mit $\mathrm{KHO}$ bis zur Verjagung des $\mathrm{NH}^{3}$ gekocht; der hierbei entstandene Niederschlag wird mit Oxalsäure behandelt, so dass die Oxalate von La, Y, Di sich niederschlagen und $\mathrm{MgO}$ in Lösung geht. Nach Glühen des Oxalatniederschlages löst man in $\mathrm{SO}^{4} \mathrm{H}^{2}$ und bildet 
Alaune, von welchen derjenige des La und eventuell Di auf dem Filter zurückbleibt, derjenige des $\mathrm{Y}$ in das Filtrat geht.

Will man nun den Theil des $\mathrm{BeO}$, welcher durch $\mathrm{HCl}$ ausgezogen und mit $\mathrm{Al}^{2} \mathrm{O}^{3}$ gesondert wurde, gewinnen, so neutralisirt man die alkalische Lösung der beiden Erden nahezu mit reinem $\mathrm{HCl}$ und schliesslich mit Essigsäure, erhitzt zum Sieden und extrahirt den erhaltenen Niederschlag nach vorheriger Reinigung von $\mathrm{SiO}^{2}$ (durch wiederholtes Auflösen, Eintrocknen und wiederholte Fällung) mittelst Ammoniumcarbonats.

Indem ich diesen vorläufigen Abschluss meiner Untersuchung der Oeffentlichkeit übergebe, bemerke ich ausdrücklich, dass ich längst Bekanntes an einem neuen Beispiele, welches die seiner Beschaffenheit entsprechende Behandlung bedarf, gezeigt, $\mathrm{m}$. W. eine Methode zur praktischen Ausbeutung der Hainstädter Ceritthone aus bekannten Daten zusammengefügt habe, und die zur Sache hinzugetretenen neuen Forschungsfragen - ob hier ein Neocerium vorliege und von welcher Zusammensetzung das lederbraune Oxyd, sowie die früher beschriebenen blauen Körper sind - anderen Interessenten einstweilen überlasse.

\section{B. Monatsbericht.}

\section{Allgemeine Chemie.}

Bestimmung der Dichte des Stiekoxydes bei $-100^{\circ}$. - Bekanntlich verwandelt sich das ungesättigte Molekül $\mathrm{NO}^{2}$, welches wir in der dissociirten Untersalpetersäure annehmen müssen, bei mässiger Abkühlunng in das gesättigte $\mathrm{N}^{2} \mathrm{O}^{4}$. Es sehien deshalb nicht unmöglich, dass auch das Molekül NO, dessen Struktar mit den allgomeinon Principien der Valenzlehre in Widerspruch steht, nichts anderes sei, als das Product der Dissociation einer unbekannten Verbindung $\mathrm{N}^{2} \mathrm{O}^{2}$. G. Daccomo und Victor Meyer suchten durch den Versuch dies zu entscheiden, erhielten aber keine Anbaltspunkte für diese Ansicht; jedenfalls existirt bei - $100^{\circ}$ eine Verbindung $\mathrm{N}^{2} \mathrm{O}^{2}$ noch nicht.

Von zwei genau gleichen Luftthermometern wurde das eine mit Luft, das andere mit Stickoxyd gefüllt. Die Sperrflüssigkeiten (Schwefelsäure) beider wurden in gleiches Niveau gestellt und nun die Luftthermometer mittelst fester Kohlensäure und Aether auf $-100^{\circ}$ abgekühlt. Die Contraction beider Gase war genau dieselbe, das Stickoxyd hatte also keine Aenderung seiner Dichte erlitten. (Ber.d.d. chem.Ges. 20, 1838.) C.J.

\section{Pharmacentische Chemie.}

Ueber Amylenhydrat. Von B. Fischer. Das neuerdings als Hypnotikum warm empfohlene Amylenhydrat ist einer der der Formel $\mathrm{C}^{5} \mathrm{H}^{12} \mathrm{O}_{\text {ent- }}$ 